\title{
EL USO DEL IPAD COMO ELEMENTO DE MEJORA DE LA COMPETENCIA DIGITAL EN LA FORMACIÓN INICIAL DE LOS MAESTROS
}

\author{
THE USE OF THE IPAD AS AN ELEMENT TO IMPROVE DIGITAL \\ COMPETENCE IN INITIAL TEACHER TRAINING
}

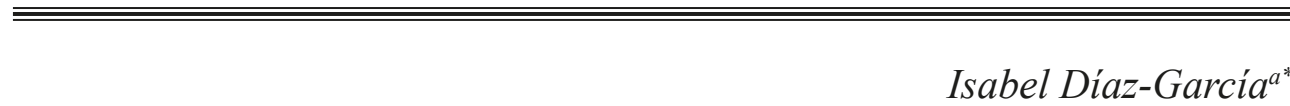

Fechas de recepción y aceptación: 14 de octubre de 2019, 8 de diciembre de 2019

Resumen: La nueva sociedad del conocimiento requiere nuevas competencias para los docentes del siglo xxi. Una de las competencias clave son las conocidas como las competencias digitales, que todo estudiante debe haber adquirido una vez acabada la enseñanza obligatoria. Por tanto, se hace necesario que los docentes estén preparados para crear los escenarios que propicien la adquisición de estas competencias. El objetivo del presente artículo es un primer acercamiento al Plan de Innovación Tecnológica en la Educación (PITE) con el fin de introducir mejoras en él. Se trata de un estudio basado en un diseño de encuesta, cuya muestra la constituyen estudiantes de las titulaciones de grado de Maestro en Educación Infantil y Primaria de la Universidad Católica de Valencia San Vicente Mártir, seleccionados mediante un muestreo no probabilístico y accidental en el que se recoge la información mediante un cuestionario. Los resultados señalan que los estudiantes están satisfechos con el plan y consideran que ha mejorado tanto su competencia digital como la digital docente. Se sugiere que se utilicen estándares y descriptores a la hora de diseñar las acciones formativas sobre la competencia digital docente en la formación inicial de los maestros y, a partir ello, se podrá evaluar y acreditar el nivel competencial y su progreso.

Palabras clave: competencia digital docente, Plan de Innovación Tecnológica en la Educación, futuros docentes, integración de las TIC, formación.

${ }^{a}$ Facultad de Filosofía y Ciencias de la Educación. Universidad de Valencia.

${ }^{*}$ Correspondencia: Departamento MIDE. Facultad de Filosofía y Ciencias de la Educación. Universidad de Valencia. Avenida Blasco Ibáñez, 30. 46010 Valencia. España.

E-mail: idiazgar@uv.es 
Abstract: The new knowledge society requires new competences for teachers of the $21 \mathrm{st}$ century. One of the key competences are those known as digital, which every student must have acquired once the compulsory education is finished. The aim of this article is a first approach to the Plan of Technological Innovation in Education (PITE) in order to introduce improvements in it. This is a study based on a survey design, whose sample is constituted by students of the degrees of Teacher of Pre-school and Primary Education at the Catholic University of Valencia, selected by a non-probabilistic and accidental sampling, in which information is collected by means of two questionnaires. The results point out that students are satisfied with the plan and that they consider both their digital competence and digital teaching have improved. It is suggested that standards and descriptors should be used when designing training actions on teacher digital competence in the initial training of teachers and, so that it can be evaluated and accredited the competence level and its progress.

Keywords: teaching digital competence, Plan of Technological Innovation in Education, pre-service teachers, ICT integration, training.

\section{INTRODUCCIÓN}

El continuo desarrollo científico y tecnológico, propio de la sociedad actual, implica una evolución constante en las habilidades requeridas para los puestos de trabajo. El sistema educativo ha de formar a ciudadanos que se enfrentan a entornos digitales caracterizados por la resolución de problemas nada rutinarios y que contienen información compleja (Claro et al., 2012; Schleicher, 2016). En esta línea encontramos que el papel del docente ha de cambiar, ya que se espera que prepare a los estudiantes para las competencias del siglo XXI (Ananiadou y Claro, 2009; Voogt y Pareja-Roblin, 2012). Entre otras, se requiere que el maestro disponga de una formación adecuada y actualizada en referencia a las nuevas tecnologías en el aula, para poder participar en la creación de recursos digitales y hacer uso de los ya existentes. Algunos organismos internacionales (Comisión Europea, 2018; Unesco, 2008) señalan la importancia del papel del maestro para los procesos de integración de las TIC en el aula. Consideran que se requiere la capacidad de los docentes para estructurar el ambiente de aprendizaje de forma no tradicional, fusionar las TIC con nuevas pedagogías, fomentando clases dinámicas en la esfera social, que estimulen la interacción cooperativa, el aprendizaje colaborativo y el trabajo en grupo. Las TIC exigen que los maestros desempeñen nuevas funciones, por lo que se hace necesario nuevos planteamientos en la forma- 
ción docente (Unesco, 2008). De la necesidad de una formación acorde con la sociedad del conocimiento y de calidad para los docentes y con el fin de mejorar el aprendizaje de los estudiantes en la adquisición de las competencias para el siglo XXI, como es la competencia digital, surge en 2012 el Plan de Cultura Digital en la Escuela (INTEF, 2012). Este plan se constituye en cinco proyectos prioritarios: la conectividad de centros escolares, la interoperabilidad y estándares, espacio "Procomún" de contenidos en abierto, el catálogo general de recursos educativos de pago: punto neutro y la competencia digital docente.

Centrando la atención en el último de ellos, se desarrolla el Marco Común de la Competencia Digital Docente (INTEF, 2017), que es un marco de referencia para el diagnóstico y la mejora de las competencias digitales del profesorado. Estas competencias digitales se definen como competencias que necesitan desarrollar los docentes del siglo XXI para la mejora de su práctica educativa y para el desarrollo profesional continuo. Este marco establece tres líneas de actuación que tienen en cuenta tanto la formación inicial como la formación continua del profesorado: $a$ ) una propuesta de marco común de referencia; $b$ ) un plan de evaluación -para poder acreditar a docentes en la competencia digital- y un impulso en paralelo de la formación docente en cuanto al nivel de competencia digital que este ha adquirido y a la que tiene que adquirir a partir de ahí.

El marco común de la competencia digital docente está formado por cinco áreas, que son: información y alfabetización informacional, comunicación y colaboración, creación de contenidos digitales, seguridad y resolución de problemas. El objetivo del área de información y alfabetización informacional es identificar, localizar, obtener, almacenar, organizar y analizar información digital, datos y contenidos digitales, evaluando su finalidad y relevancia para las tareas docentes. El objetivo del área de comunicación y colaboración es el de comunicar en entornos digitales, compartir recursos a través de herramientas en línea, conectar y colaborar con otros a través de herramientas digitales, interactuar y participar en comunidades y redes; conciencia intercultural. En cuanto al objetivo del área de creación de contenidos digitales, consiste en crear y editar contenidos digitales nuevos, integrar y reelaborar conocimientos y contenidos previos, realizar producciones artísticas, contenidos multimedia y programación informática, saber aplicar los derechos de propiedad 
intelectual y las licencias de uso. La protección de información y datos personales, protección de la identidad digital, de los contenidos digitales, medidas de seguridad y uso responsable y seguro de la tecnología, es el objetivo del área de seguridad. Y, por último, desde el área de resolución de problemas se pretende identificar necesidades de uso de recursos digitales, tomar decisiones informadas sobre las herramientas digitales más apropiadas según el propósito o la necesidad, resolver problemas conceptuales a través de medios digitales, usar las tecnologías de forma creativa, resolver problemas técnicos, actualizar su propia competencia y la de otros (INTEF, 2017). En cada una de estas áreas, se encuentran un total de 21 competencias.

Además, se establecen 6 niveles competenciales progresivos de dominio por cada área. Dentro de cada nivel aparecen sus descriptores. La estructura está diseñada para identificar el nivel de competencia digital de un docente en cada una de las 21 competencias, estableciendo, así, un nivel progresivo de desarrollo y autonomía que parte del nivel A1 y continúa hasta el nivel máximo, $\mathrm{C} 2$.

Por otro lado, y dentro del currículum de enseñanza obligatoria, se contemplan siete competencias claves que todo estudiante debe haber adquirido una vez acabada la etapa. Si los maestros no tienen adquiridas las competencias claves ni tienen estrategias para crear entornos de aprendizaje donde los estudiantes las adquieran, difícilmente van a poder acabar la enseñanza obligatoria con la consecución de estas competencias. Entre las siete competencias claves, encontramos la digital, que dentro del contexto escolar constituye el conjunto de conocimientos y habilidades que permiten dominar distintos recursos tecnológicos para su utilización en sus diversas tareas académicas, de forma ética, segura y responsable (Almerich, Díaz-García, Cebrián-Cifuentes y Suárez-Rodríguez, 2018). De ahí la necesidad de formar a los futuros maestros en las competencias digitales. De esta necesidad nace en el curso 2014/15 el Plan de Innovación Tecnológica en la Educación (PITE) en la Universidad Católica de Valencia San Vicente Mártir (UCV). Este plan consiste en la integración del uso del iPad en la formación inicial de los futuros maestros de infantil y primaria de la UCV con el fin de que adquieran la competencia digital docente.

El Plan de Innovación Tecnológica en la Educación (2016) posee tres líneas de actuación: dotación de infraestructuras (de iPads para profesores y 
estudiantes, de puntos de acceso en las aulas, de adaptadores para poder proyectar el iPad en las aulas, etc.), formación de los miembros de la comunidad universitaria implicados (profesores y estudiantes) y evaluación del plan con el fin de incluir acciones de mejoras de este.

La formación que reciben los profesores de la Facultad de Magisterio, consta de dos partes: primero, una formación inicial para manejar el iPad y poderlo conectar en el aula para posteriormente llevar a cabo una formación continuada en metodologías docentes a través de aplicaciones de la tableta. Esta formación tiene tres líneas de contenido: el uso básico del iPad y las aplicaciones docentes, el iPad para uso docente y el uso metodológico a través del iPad. Al profesorado se le va formando según se incorpora al plan. Cada curso se van incorporando nuevos profesores hasta llegar a la totalidad del claustro.

En cuanto a la formación que reciben los estudiantes pertenecientes al grado de Maestro de Educación Infantil y/o Primaria cabe señalar que se integra dentro de la materia de prácticum-TIC. Su inicio se sitúa una semana antes de la entrega del dispositivo. La formación inicial consta de 8 sesiones de 2 horas cada una y en ella se incluyen: el manejo del iPad, buenas prácticas (uso adecuado del iPad y de internet); el uso del campusUCVnet y Drive y Google Presentaciones, Form, Docs, Adobe (edición de PDF); el manejo de las aplicaciones de Apple (iMovie, Keynote, etc.), el manejo de aplicaciones para realizar mapas conceptuales como Mindomo. Por otro lado, y como uso para la docencia, los estudiantes realizan formación basada en proyectos, tales como la creación de actividades didácticas con QR y la creación de cuentos con Sketchbooks y Skitch. En cursos sucesivos se centra la formación en aplicaciones diseñadas para su uso en los procesos de enseñanzaaprendizaje, en este caso, como futuros docentes (Socrative, Educanon y Apple\&education, etc.)

Por último, el plan consta de un tercer pilar que consiste en la evaluación de dicho plan como proceso de mejora. Se ha realizado una dotación de las infraestructuras necesarias, se ha formado a los agentes implicados pero no se conoce el uso que hacen de él como herramienta para su desempeño docente ni para la realización de las tareas formativas de los estudiantes ni si este ha supuesto un incremento en la competencia digital de los participantes. Dicho todo esto, se hace necesario realizar un primer diagnóstico sobre el uso, el conocimiento y las actitudes frente a las TIC dentro del contexto educativo 
que ha supuesto la introducción de la herramienta en la facultad, así como establecer el nivel de competencia digital docente de los profesores y estudiantes -futuros docentes- que participan en el plan. Esto va a permitir conocer el estado en el que se encuentra dicho plan y, a partir de ahí, introducir acciones de mejora basadas en evidencias recogidas de un modo sistemático.

El presente trabajo forma parte de este último pilar del Plan de Innovación Tecnológica en la Educación de la Facultad de Magisterio y Ciencias de la Educación de la Universidad Católica de Valencia San Vicente Mártir.

\section{MÉtodo}

El objetivo de este trabajo es conocer en qué momento se encuentra el Plan de Innovación Tecnológica en la Educación desde la perspectiva de los estudiantes de la Universidad Católica de Valencia con el fin de poder incorporar acciones de mejora en dicho plan. Para ello, vamos a indagar sobre el grado de satisfacción de los estudiantes con el PITE, sobre la mejora del nivel de competencia digital y del de la competencia digital docente desde que el plan está en marcha, sobre el uso que hacen del dispositivo tanto para cuestiones académicas como personales y, finalmente, sobre las actitudes que tienen estos estudiantes hacia los recursos tecnológicos.

Este estudio se basa en un diseño de encuesta, cuya población la constituye los estudiantes de las titulaciones de grado de Maestro de Educación Primaria e Infantil de la Universidad Católica de Valencia San Vicente Mártir.

La muestra, seleccionada mediante un muestreo no probabilístico accidental, está constituida por 348 estudiantes del grado de Maestro de Educación Primaria (38,1 \%), del grado de Maestro de Educación Infantil (10,3\%), del doble grado de Maestro de Educación Infantil y Primaria (46,4 \%) y del doble grado de Maestro de Educación Primaria y Prica ${ }^{1}$ (5,2 \%) de la Universidad de Católica de Valencia. El 22,6 \% son hombres y el 77,4 \%, mujeres, siendo la edad media de los participantes de 21.4 años y un rango que va de 18 a 42 años. En cuanto al curso al que pertenecen, el 20,3 \% son de primero, el

\footnotetext{
${ }^{1}$ Hace referencia al grado en Ciencias de la Actividad Física y del Deporte.
} 
$42,4 \%$ de segundo, el 36,7 \% de tercero y el 0,6 \% de cuarto curso. El $100 \%$ de los estudiantes dispone de un iPad nuevo para su uso académico y como herramienta formativa para el futuro docente. Al 24,6\% de los estudiantes se le entregó el iPad en el curso académico 2014/15: el 34,5 \% lo recibió en el curso 2015/16 y el 40,8 \% durante el curso académico 2016/17.

En relación con el instrumento de recogida de la información, se han utilizado un cuestionario, que ha sido diseñado para tal fin, que recoge información sobre diversos apartados relativos a: las características sociodemográficas de los estudiantes (7 ítems), el grado de satisfacción de los estudiantes con el plan (3 ítems), las limitaciones sobre las infraestructuras proporcionadas (3 ítems), el grado de formación de los estudiantes (4 ítems), el nivel de conocimiento acerca del uso del iPad (1 ítem), el tiempo de uso (personalacadémico) que hacen los estudiantes del iPad (2 ítems), la frecuencia de uso de diferentes recursos digitales con el iPad (16 ítems) y la percepción sobre la posible mejora de la competencia digital y la competencia digital docente de los futuros maestros (2 ítems). Se ha utilizado una escala tipo Likert de 5 puntos en la mayoría de las dimensiones estudiadas. En cuanto a la fiabilidad de la dimensión de frecuencia de uso (personal y académico) de diferentes aplicaciones del iPad se ha obtenido $\alpha$ de Cronbach $=.83$.

En cuanto al procedimiento, cabe señalar que los cuestionarios se han recogido en formato online durante el año 2017. Los análisis han sido obtenidos mediante el programa SPSS 24.

\section{REsultados}

Dado que es un primer acercamiento, los análisis realizados son estadísticos descriptivos y constituyen frecuencias de las distintas dimensiones que conforman el cuestionario. En segundo lugar, se ha llevado a cabo un análisis de ANOVA de un factor con el fin de determinar si existen diferencias en cuanto a la frecuencia de uso del iPad para cuestiones académicas, en diferentes recursos tecnológicos a partir de la antigüedad de los estudiantes en el plan. 


\subsection{Acercamiento a la opinión y dimensiones generales (infraestructuras,} formación, uso, etc.) de los estudiantes implicados en el Plan de Innovación Tecnológica en la Educación (PITE)

A continuación se describen cada uno de los aspectos generales más relevantes:

En cuanto a la opinión y satisfacción de los futuros maestros sobre el Plan de Innovación Tecnológica en la Educación, el 71.9 \% afirma que está muy satisfecho con el Plan de Innovación Tecnológica en la Educación, el 20,1 \% que está satisfecho sin más, el 4,9\% que no y el 3,2\% dice estar muy insatisfecho con el plan, siendo la media de un 7,3 sobre 10. El 87,4 \% considera que el PITE debería generalizarse a otras titulaciones, frente al 12,6\% que considera que no debería hacerse. Además, el 95,1 \% de los estudiantes universitarios consideran que el PITE es necesario y que no debería eliminarse en un futuro.

En cuanto a las infraestructuras y la calidad de la conexión de la universidad donde se imparten las titulaciones, el 53,8 \% afirma que es adecuada, frente al 39,8 \% que señala que no lo es. En lo que se refiere al acceso a la red, el 36,1 \% afirma que es sencilla y fácil, frente al $47 \%$, que señala que no es ni sencilla ni fácil.

En cuanto a la formación recibida sobre el uso del iPad, el $86.2 \%$ dice haber recibido algún tipo de formación sobre el uso del iPad, frente al 13.8\% que afirma no haberla recibido. El 72,4\% de los estudiantes universitarios afirman que la formación que han recibido sobre el uso del iPad ha sido en la propia facultad, frente al $0,7 \%$ que la ha recibido de fuera de ella, siendo el $23,2 \%$ los estudiantes que afirman que han recibido dicha formación tanto en la universidad como fuera de esta. Poco más de la mitad de los estudiantes $(50,2 \%)$ considera que necesita más formación sobre el uso del iPad frente al 49,8 \% que no lo considera así. Lo que sí que destacan $(84,1 \%)$ es la necesidad de recibir más formación de la herramienta (iPad) para su uso docente.

En cuanto a las horas diarias, cabe señalar que la media para el uso personal es de 1.85 (que supone menos de una hora diaria), frente al 2.32 (entre una y tres horas diarias) para el uso del iPad en cuestiones académicas. Por lo que el tiempo que destinan para el uso académico es mayor que el que dedican 
para utilizarlo para asuntos personales. En concreto, en relación con el tiempo que usan el iPad para cuestiones personales, el 40,7\% de los estudiantes universitarios afirman usarlo menos de una hora, el 40,7 \% lo hacen entre una hora y tres, el 11,7 \% lo hacen entre tres y seis horas y el 6,9\% afirman usarlo más de seis horas diarias. Por otro lado, en cuanto al uso académico que hacen del iPad, el 18,9\% dicen usarlo menos de una hora diaria, el 39,8 \% lo hacen entre una y tres horas al día, el 32,1\% lo usan para cuestiones académicas entre tres y seis horas diarias y, por último, el 9,2\% dice usarlo más de seis horas diarias para tareas académicas (véase gráfico 1).

\section{GRÁFICO 1}

Número de horas diarias que utilizas el iPad para uso personal y académico

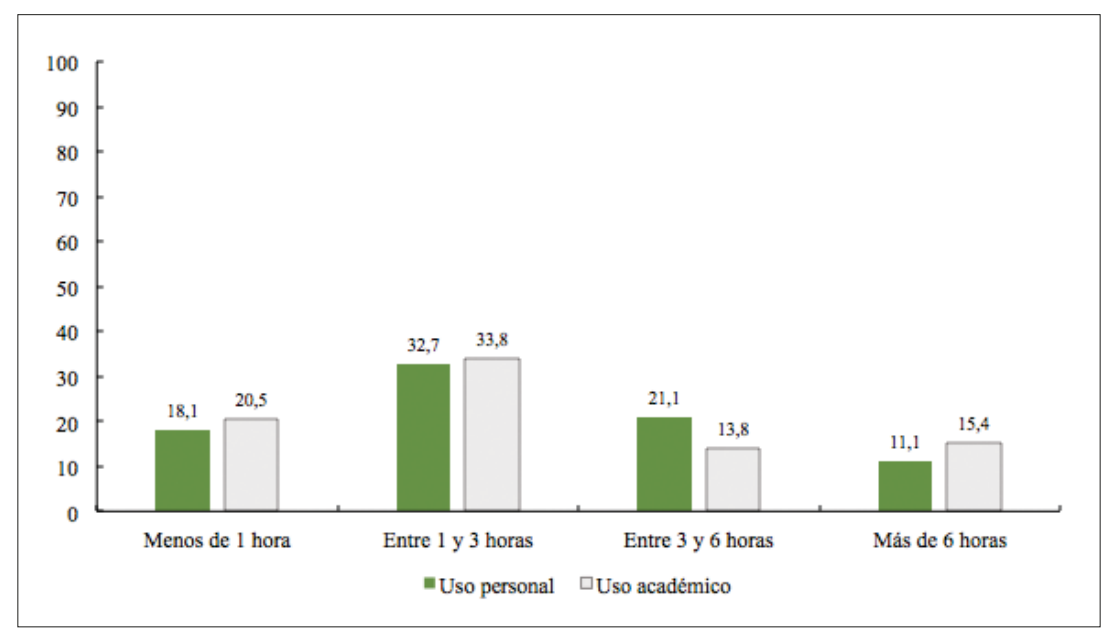

Sobre el grado de conocimiento del manejo del iPad, el $8 \%$ de los estudiantes se consideran principiantes, el 43,6 \% considera que tienen un conocimiento intermedio, el $42,1 \%$ dicen poseer un conocimiento avanzado sobre el uso de iPad y es el 6,3\% el que se considera que tiene un conocimiento de experto (véase gráfico 2). Por otro lado, el 83,7\% consideran que con el manejo del iPad ha mejorado su competencia digital, frente al 16,3\% que no. El 79,4 \% considera que su competencia digital docente ha mejorado con el uso del iPad y el 20,6\% consideran que no lo ha hecho. 


\section{GRÁFICO 2}

Grado de conocimiento que consideras que tienes sobre el uso del iPad

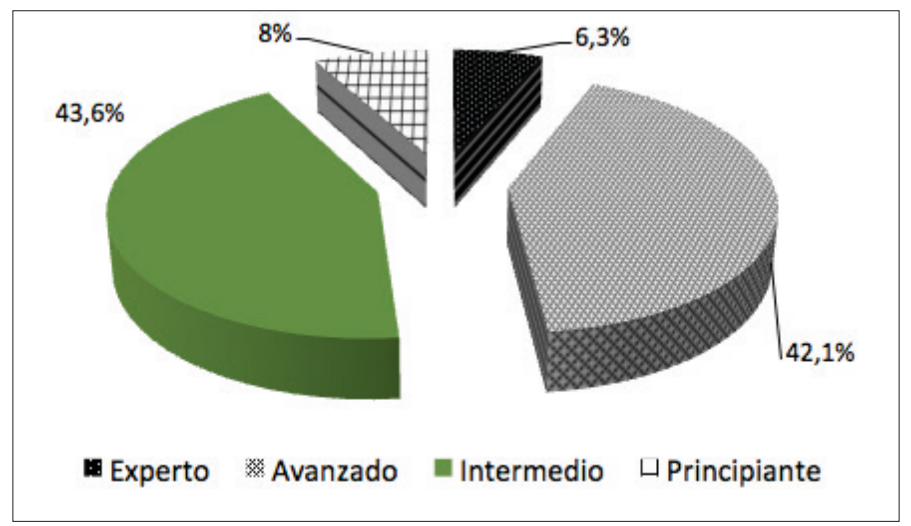

Por otro lado, el 83,7 \% consideran que con el manejo del iPad ha mejorado su competencia digital, frente al 16,3\% que no. El 79,4\% consideran que su competencia digital docente ha mejorado con el uso del iPad, y el 20,6 \% que no lo han hecho.

\section{GRÁFICO 3}

Consideración sobre la mejora de la competencia digital y digital docente tras el Plan de Innovación Tecnológica en Educación

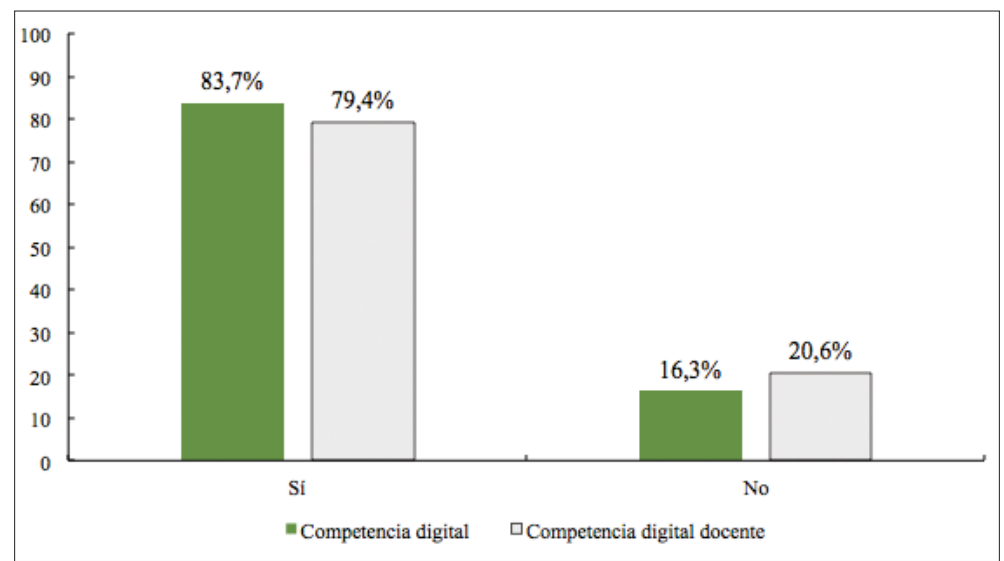


Respecto a la frecuencia de uso del iPad para los servicios generales de internet (véase tabla 1), las medias se encuentran en torno a una frecuencia de "semanalmente", siendo el uso del navegador la media más elevada y la de las redes sociales la media más baja, en torno a una frecuencia mensual (siendo $1=$ nada, 2 = cada 3 meses, $3=$ mensualmente, $4=$ semanalmente, $5=$ diariamente). En cuanto a la frecuencia de uso de recursos tecnológicos para tareas académicas, encontramos que lo que más se usa son los buscadores, seguido por los documentos de Google, las presentaciones y el procesador de textos, que se sitúan en una media de alrededor de 4. Las que menos frecuencia de uso tienen son Popplet/Mindomo, aplicaciones online para elaborar mapas conceptuales, cuya frecuencia se sitúa en una media de 2.84 (siendo $1=$ nunca, 2 = poco (una vez en el curso) 3 = algo (una vez al semestre), $4=$ mucho (al menos dos veces al mes, $5=$ siempre (todas las semanas).

\section{TABLA 1}

Descriptivos de la frecuencia de uso del iPad para el manejo de recursos tecnológicos

\begin{tabular}{|l|c|c|}
\hline \multicolumn{1}{|c|}{ Frecuencia de uso de servicios generales de internet } & Media & Desv. típ. \\
\hline Navegador & 4,4 & 0,73 \\
\hline Plataforma UCV & 4,37 & 0,761 \\
\hline Drive, Dropbox, etc. & 4,01 & 0,994 \\
\hline Correo electrónico & 4,31 & 0,793 \\
\hline Redes sociales & 3,52 & 1,347 \\
\hline Frecuencia de uso de recursos tecnológicos para tareas académicas & & \\
\hline Buscadores & 4,56 &, 769 \\
\hline iMovie & 3,15 & 1,184 \\
\hline Procesador de texto & 3,91 & 1,294 \\
\hline Presentaciones & 3,99 &, 981 \\
\hline Edición de vídeo/audio/imagen & 3,43 & 1,253 \\
\hline Documentos de Google (Doc, Presentaciones, Form, etc.) & 4,2 & 1,085 \\
\hline Popplet, Mindomo, etc. (mapas conceptuales) & 2,84 & 1,367 \\
\hline Adobe Reader & 3,93 & 1,281 \\
\hline Kahoot (crearlo) & 3,04 & 1,385 \\
\hline
\end{tabular}




\subsection{Acercamiento a la opinión y dimensiones generales (infraestructuras,} formación, uso, etc.) de los estudiantes implicados en el Plan de Innovación Tecnológica en la educación (PITE)

Con el fin de determinar si existen diferencias en cuanto a la frecuencia de uso del iPad para cuestiones académicas, en diferentes recursos tecnológicos, a partir de la antigüedad de los estudiantes en el plan se ha realizado el análisis de ANOVA de un factor.

La mayor frecuencia de uso se produce en los tres grupos de antigüedad -véase gráfico 4- en los "buscadores", seguido por los diferentes documentos que proporciona Google Drive; ambos recursos se sitúan por encima de una media de 4, que viene a ser una frecuencia de uso semanal. En cuanto a los recursos que obtienen una menor media de frecuencia de uso - por debajo de 3 , que es una frecuencia de una vez en el curso--, se encuentran Kahoot y el uso de herramientas para realizar mapas conceptuales (Mindomo y Popplet).

\section{GRÁFICO 4}

Puntuaciones medias de diferentes recursos tecnológicos en función de la antigüedad en el PITE

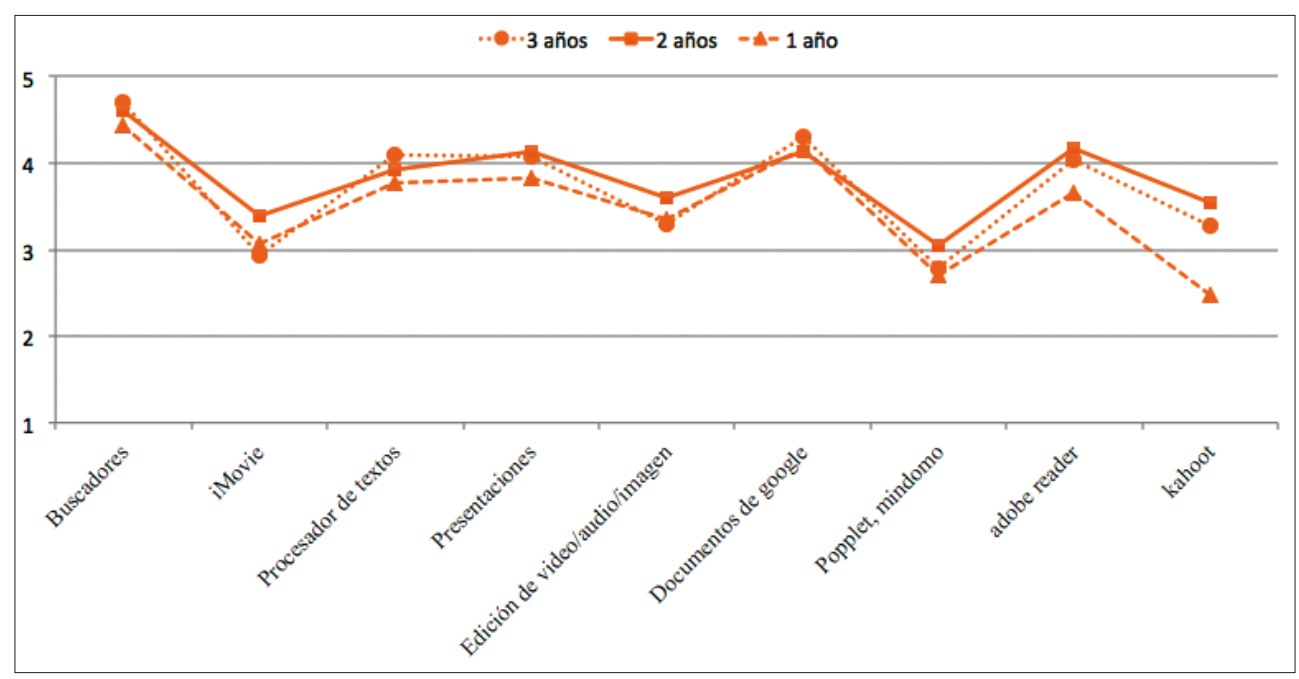


Cabe señalar que en cuanto a las puntuaciones medias, en función de la antigüedad que tienen los estudiantes, se produce una tendencia que se repite en cada uno de los recursos tecnológicos analizados, consistente en una menor frecuencia de uso en función de una menor antigüedad, es decir, el llevar únicamente un año en el PITE obtiene menor frecuencia de uso a rasgos generales que entre los que llevan dos años o tres.

A nivel univariado, tras los ANOVAS realizados, se han encontrado diferencias estadísticamente significativas en "buscadores", "iMovie", "presentaciones", “Adobe Reader" y "Kahoot” -véase tabla 2-.

TABLA 2

Descriptivos en función del año de la antigüedad de los estudiantes en el plan y ANOVA de la frecuencia de uso de los recursos tecnológicos para cuestiones académicas como futuros docentes

\begin{tabular}{|c|c|c|c|c|c|}
\hline $\begin{array}{l}\text { Frecuencia de uso académico de } \\
\text { los recursos tecnológicos }\end{array}$ & Grupo & Media & $\begin{array}{l}\text { Desviación } \\
\quad \text { típica }\end{array}$ & $F$ & Sig. \\
\hline \multirow{3}{*}{ Buscadores } & 3 años & 4,70 &, 595 & \multirow{3}{*}{3,197} & \multirow{3}{*}{, 042} \\
\hline & 2 años & 4,60 & ,703 & & \\
\hline & 1 año & 4,44 & 895 & & \\
\hline \multirow{3}{*}{ iMovie } & 3 años & 2,93 & 1,196 & \multirow{3}{*}{4,458} & \multirow{3}{*}{,012 } \\
\hline & 2 años & 3,39 & 1,040 & & \\
\hline & 1 año & 3,06 & 1,256 & & \\
\hline \multirow{3}{*}{ Procesador de texto } & 3 años & 4,09 & 1,164 & \multirow{3}{*}{1,742} & \multirow{3}{*}{, 177} \\
\hline & 2 años & 3,93 & 1,282 & & \\
\hline & 1 año & 3,77 & 1,372 & & \\
\hline \multirow{3}{*}{ Presentaciones } & 3 años & 4,07 & 992 & \multirow{3}{*}{3,810} & \multirow{3}{*}{,023 } \\
\hline & 2 años & 4,14 &, 823 & & \\
\hline & 1 año & 3,82 & 1,074 & & \\
\hline \multirow{3}{*}{ Edición de vídeo/audio/imagen } & 3 años & 3,30 & 1,329 & \multirow{3}{*}{1,784} & \multirow{3}{*}{, 169} \\
\hline & 2 años & 3,60 & 1,162 & & \\
\hline & 1 año & 3,36 & 1,274 & & \\
\hline \multirow{3}{*}{$\begin{array}{l}\text { Documentos de Google (doc, pre- } \\
\text { sentaciones, form, etc.) }\end{array}$} & 3 años & 4,31 & 1,032 & \multirow{3}{*}{, 731} & \multirow{3}{*}{,482 } \\
\hline & 2 años & 4,13 & 1,130 & & \\
\hline & 1 año & 4,18 & 1,080 & & \\
\hline
\end{tabular}




\begin{tabular}{|c|c|c|c|c|c|}
\hline $\begin{array}{l}\text { Frecuencia de uso académico de } \\
\text { los recursos tecnológicos }\end{array}$ & Grupo & Media & $\begin{array}{l}\text { Desviación } \\
\text { típica }\end{array}$ & $F$ & Sig. \\
\hline \multirow{3}{*}{$\begin{array}{l}\text { Popplet, Mindomo, etc. (mapas } \\
\text { conceptuales) }\end{array}$} & 3 años & 2,79 & 1,398 & \multirow{3}{*}{2,263} & \multirow{3}{*}{, 106 } \\
\hline & 2 años & 3,05 & 1,269 & & \\
\hline & 1 año & 2,70 & 1,414 & & \\
\hline \multirow{3}{*}{ Adobe Reader } & 3 años & 4,03 & 1,202 & \multirow{3}{*}{5,903} & \multirow{3}{*}{, 003} \\
\hline & 2 años & 4,18 & 1,167 & & \\
\hline & 1 año & 3,66 & 1,373 & & \\
\hline \multirow{3}{*}{ Kahoot (crearlo) } & 3 años & 3,28 & 1,252 & \multirow{3}{*}{23,893} & \multirow{3}{*}{,000 } \\
\hline & 2 años & 3,54 & 1,137 & & \\
\hline & 1 año & 2,47 & 1,452 & & \\
\hline
\end{tabular}

Tras realizar el DMS para conocer en qué sentido se producen las diferencias, podemos señalar que en el caso de los "buscadores" es entre los que tienen tres años de antigüedad en el plan y los que llevan un año, a favor de los primeros. En el caso de "iMovie" encontramos diferencias significativas en la frecuencia de uso por antigüedad entre los que tienen dos años de antigüedad en el plan y los que participan por primera vez en el plan, a favor de los primeros. En lo que se refiere a las "presentaciones" existen diferencias significativas en la frecuencia de uso en función de la antigüedad en el plan, entre los de segundo año en el plan y los de primer año, a favor de los primeros. En cuanto a las diferencias encontradas en la frecuencia de uso del "Adobe Reader", cabe señalar que las diferencias encontradas se dan entre los que llevan más tiempo en el plan y los que menos tiempo llevan, a favor de los primeros, y, por otro lado, entre los que llevan dos años en el plan y los que llevan únicamente uno, a favor de los primeros; por último, los que llevan tres años se diferencian con respecto a los que llevan dos años, a favor de los menos antiguos. Para finalizar, se han encontrado diferencias significativas en la frecuencia de uso del Kahoot entre los estudiantes que tienen menos experiencia en el plan y los que más tienen, y esta es a favor de los más antiguos, es decir, los que llevan más tiempo en el PITE usan con mayor frecuencia Kahoot que los que llevan menos tiempo. 


\section{CONCLUSIONES}

El presente trabajo permite hacerse una idea general sobre la opinión de los estudiantes con respecto al PITE. Se puede decir que la mayor parte de los estudiantes están satisfechos con el Plan de Innovación Tecnológica en la Educación y que la mayoría piensa que debería generalizarse a otras titulaciones. Con respecto a la competencia digital de los estudiantes, estos afirman que ha mejorado con el plan; además, señalan que también ha mejorado su competencia digital docente. Habría que mejorar el instrumento de recogida de información, ya que con el planteamiento actual no se tienen elementos que puedan ayudar a definir las posibles mejoras que harían que los estudiantes estuviesen más satisfechos con el plan. Es decir, habría que incluir un apartado en el que nos señalarán cosas que se pueden mejorar. Esto puede hacerse mediante una entrevista semiestructurada a estudiantes clave y hacer cuestiones que permitan la mejora. Por otro lado, habría que mejorar la conexión de internet de que dispone la facultad. Las infraestructuras necesarias son básicas para que el plan pueda llevarse a cabo en condiciones óptimas y la mejora de la conexión es clave en este sentido. Por otro orden de cuestiones, la triangulación de la información se realiza a través de la información de los profesores implicados en el plan y que han participado en la primera fase del estudio a través de otro cuestionario elaborado para tal efecto, con dimensiones similares a las estudiadas en los estudiantes.

Cabe señalar que los resultados obtenidos no nos permiten comprobar el nivel de los futuros maestros en cuanto a la competencia digital. Por ello, se hace especial hincapié en futuras líneas de indagación, que pasan por los siguientes aspectos: a) diseñar la formación a lo largo de los cursos basándose en los estándares y descriptores definidos en el marco común de la competencias digitales docentes (INTEF, 2017) y sobre la base de las 5 áreas y sus 21 competencias. Se hace necesario establecer desde la propia facultad un nivel mínimo que alcanzar para, a partir de este, establecer y diseñar las acciones formativas necesarias para que los futuros maestros cuando acaben el grado hayan adquirido el nivel de competencia definido. Y b) es de gran relevancia que existan estos estándares y descriptores ya consensuados para que los futuros docentes puedan acreditar y establecer el nivel que han adquirido y así seguir progresando en la adquisición de niveles superiores de la competencia 
digital docente, de ahí que desde la facultad se trabaje en la evaluación y acreditación de las competencias digitales docentes.

\section{BiBLIOGRAFÍA}

Almerich, G., Díaz-García, I., Cebrián-Cifuentes, S. y SuÁrez-Rodríguez J. (2018). Estructura dimensional de las competencias del siglo XXI en alumnado universitario de educación. RELIEVE 24(1), art. 5. doi: http://doi. org/10.7203/relieve.24.1.12548.

Ananiadou, K. y Claro, M. (2009). 21st century skills and competences for new millennium learners in OECD countries. Organisation for Economic Cooperation and Development. EDU Working paper 41. doi: https://doi. org/10.1787/218525261154.

Arras, A. M. D. G., Torres, C. A. y García-Valcárcel, A. (2011). Competencias en Tecnologías de Información y Comunicación (TIC) de los estudiantes universitarios. Revista latina de comunicación social 66, 130-152. doi: https://doi.org/10.4185/RLCS-66-2011-927-130-152.

Claro, M., Preiss, D. D., San Martín, E., Jara, I., Hinostroza, J. E., Valenzuela, S., Cortés, F. y Nussbaum, M. (2012). Assessment of 21 st century ICT skills in Chile: Test design and results from high school level students. Computers \& Education 59(3), 1042-1053. doi: https://doi.org/10.1016/j. compedu.2012.04.004.

Comisión Europea (2018). ANEXO de la Propuesta de Recomendación del Consejo relativa a las competencias clave para el aprendizaje permanente. Recuperado de: https://eur-lex.europa.eu/resource.html?uri=cellar:395443f6fb6d-11e7-b8f5-01aa75ed71a1.0013.02/DOC_2\&format=PDF.

Díaz-García, I., Cebrián-Cifuentes, S. y Fuster-Palacios, I. (2016). Las competencias en TIC de estudiantes universitarios del ámbito de la educación y su relación con las estrategias de aprendizaje. RELIEVE 22(1). doi: http://doi.org/0.7203/relieve.22.1.8159.

INTEF (2017). Marco Común de Competencia Digital Docente - Octubre 2017. Recuperado de: https://aprende.intef.es/sites/default/files/ 2018-05/2017_1020_Marco-Com\%C3\%BAn-de-Competencia-Digital -Docente.pdf. 
Schleicher, A. (2016). Desafíos para PISA. RELIEVE 22(1). doi: http://doi. org/10.7203/relieve.22.1.8429.

Voogt, J. y PAReja-Roblin, N. (2012). A comparative analysis of international frameworks for 21 st century competences: Implications for national curriculum policies. Journal of Curriculum Studies 44(3), 299-321. doi: https://doi.org/10.1080/00220272.2012.668938. 
